\title{
Factores explicativos de la práctica de voluntariado corporativo en España
}

\author{
Alicia Guerra Guerra \\ Universidad de Extremadura \\ aguerra@unex.es
}

Resumen: Entendemos por voluntariado corporativo el practicado por los empleados bajo el liderazgo y la organización de la empresa. Se trata de una herramienta de la responsabilidad social de la empresa que permite a esta transmitir confianza ante la sociedad, al tiempo que genera importantes beneficios para ella, sus empleados y la comunidad que lo recibe. Estos argumentos, junto al llamamiento de la UE y del gobierno español a profundizar en el estudio de esta forma de voluntariado dada la insuficiencia de investigaciones en este campo, la debilidad del voluntariado corporativo en España respecto a países de su nivel socioeconómico y el deseo de su estímulo por parte de los poderes politicos españoles, motivan este trabajo. Su objetivo es determinar los factores que pueden explicar la práctica de voluntariado corporativo en España. Para ello se encuesta a directivos responsables del voluntariado de sus empresas acerca de las variables determinantes del voluntariado corporativo que apuntan las investigaciones previas. Los resultados confirman que todas ellas, con diversa intensidad, influyen en la decisión de practicar voluntariado por parte de las empresas. Entre ellas destacan el becho de que la empresa pasa a ser considerada como socialmente responsable, seguido de los efectos beneficiosos que genera en el personal de la empresa (creadores de un vinculo emocional con la empresa tal que les hace sentir orgullo de pertenencia a ella) $y$ la mejora de las relaciones de la empresa con la comunidad.

Palabras clave: voluntariado corporativo, responsabilidad social corporativa, factores del voluntariado corporativo. 


\section{Motivating Factors in Corporate Volunteering in Spain}

Abstract: Corporate volunteering refers to voluntary work carried out by employees under the leadership and organization of a company. It is a form of corporate social responsibility used by a company to transmit a positive image to society whilst also generating significant benefits for the company, its employees and the community that is the recipient of the voluntary work. This study, therefore, focuses on corporate volunteering, particularly given the calls by both the EU and the Spanish government for greater research into this form of volunteering, the low rate of corporate volunteering in Spain compared to countries of a similar socioeconomic level, and the desire among Spanish politicians to stimulate this practice. To this end, we surveyed company managers responsible for corporate volunteering to determine which of the factors highlighted by previous research most influenced their decision to engage in the practice. The results confirm that with varying intensity all of these factors influence companies' decisions to carry out corporate volunteer work. The most important factors are that the company is perceived to be socially responsible, that corporate volunteering has beneficial effects on company employees (whereby an emotional bond is formed with the company that makes them feel proud to belong to it) and that relations between the company and the community are improved.

Keywords: employee volunteering, corporate social responsibility, determining factors of employee volunteering. 


\section{Introducción}

El voluntariado corporativo (VC) o voluntariado de empresa se enmarca dentro de la responsabilidad social de las empresas (RSE), de la que constituye una de sus facetas (Comisión Europea, 2011a). Esto es así debido a que, si la RSE se define como el compromiso voluntario de las empresas con el desarrollo de la sociedad y la conservación del medio ambiente (AECA, 2004ㄹ), el VC alude al conjunto de acciones realizadas por iniciativa de la empresa, y en muchos casos impulsadas por ella, y sus trabajadores destinadas a cooperar con la sociedad y el medio ambiente, en las que intervienen los empleados y jubilados de la empresa así como, en no pocas ocasiones, sus familiares y amigos, liderados todos por la empresa en su afán de trasladar su cultura y valores no solo al interior -empleados-, sino también al exterior de la empresa (Guardia et alii, 2006). Pretende, así pues, incentivar y encauzar el potencial humano y profesional de una empresa hacia la satisfacción de necesidades sociales y ecológicas, compartiendo con la RSE su voluntariedad y gratuidad (León, 2010). Esta actividad implica normalmente ofrecer tiempo y experiencia a entidades no lucrativas y se realiza bien durante el tiempo libre del empleado, bien durante su horario de trabajo.

A modo de ejemplo, por VC estamos aludiendo a iniciativas tales como el conjunto de empleados voluntarios de Telefónica integrados en el Programa Proniño, con el que esta empresa pretende la erradicación de la mano de obra infantil en Hispanoamérica (Telefónica, 2011); o las reforestaciones llevadas a cabo por sus empleados voluntarios en el corredor ecológico Ardilla - que aspira a conectar los bosques españoles-, actividad incluida en el Programa Plantemos para el Planeta (vinculado a Naciones Unidas).

Las acciones de VC están emergiendo con fuerza en las empresas como una herramienta importante que demuestra que practican RSE (Burnes y Gonyea, 2005) y que transmiten fiabilidad y confianza de la empresa ante la sociedad (Muthuri et alii, 2009). Esta fortaleza procede de que, además de aportar valores personales y de otra índole muy variada a los empleados (Dávila, 2010), así como beneficios a la comunidad de destino de tales acciones (Peterson, 2004a; Meijs y Roza, 2010; $\left.C E x S^{2}, 2010\right)$, beneficia en última instancia a la empresa que lo practica. Es más, encierra aún un tremendo potencial de mejora para la empresa; así, si bien los directivos en Estados Unidos perciben las ventajas que reporta a la

1 Entenderemos por grupos de interés o stakeholders todas aquellas personas o colectivos que, de un modo u otro, se ven afectados por la vida de la empresa. La RSE se materializa en el cumplimiento por parte de la empresa de las expectativas de sus grupos de interés respecto de ella.

2 Club de Excelencia en Sostenibilidad y Fundación Adecco. 
empresa a corto y largo plazo, a menudo no aplican a sus programas de voluntariado una perspectiva orientada al negocio (Deloitte, 2010).

Consciente de esta valía, y tras celebrar 2011 como Año Europeo del Voluntariado, la Comisión Europea (2011b) lanza, entre otros desafíos para el impulso del voluntariado - incluido el VC-, los de combatir su escaso reconocimiento social y de elaborar más estudios a fin de conocerlo en una profundidad tal que oriente la eficacia de las políticas públicas. En el ámbito de la iniciativa privada, el programa internacional de VC ENGAGE, promovido por la organización empresarial inglesa Business in the Community, se convierte en Europa en el referente de excelencia en el estímulo de este voluntariado (Lemonche, 2011).

España se ha incorporado relativamente tarde a la demanda mundial de ética empresarial (Vogel, 2005), ya que comenzó sus actividades en RSE hacia fines de los años noventa del pasado siglo (Aldámiz-Echevarría y Aguirre, 2010). En consecuencia, y pese a su avance constante al ser incorporado por las empresas como parte de sus programas de RSE (González et alii, 2008), España muestra una debilidad relativa en VC en relación con países de nuestro entorno y similar nivel económico y social, especialmente con EE.UU y el Reino Unido (Tuffrey, 2003; Dávila, 2010); de ahí que el Ministerio de Sanidad, Política Social e Igualdad (2011) considerase al VC como clave en su política de impulso del voluntariado.

La crisis económica puede estar lastrando aún más esta convergencia del VC en nuestro país: el 65\% de las empresas con más de 500 empleados declaraban en 2010 realizar actividades de $\mathrm{VC}$, pero solo el $57 \%$ de las empresas de este tamaño lo practicaban en 2011; aluden a otras prioridades en la empresa como el argumento principal, a gran distancia del resto, para no desarrollar acciones de este tipo (Observatorio de VC, 2010, 2011). Sorprende comprobar cómo el segundo motivo de no practicar VC es la falta de conocimiento del mismo por parte de la empresa (2010) o el hecho de que no se lo haya planteado (2011).

La Estrategia Estatal del Voluntariado (2010-2014) —fundamentada en la estrategia de UE - se hace eco de esta realidad y, tras reconocer que se trata de una de las vertientes del voluntariado actualmente en desarrollo y con una importante proyección en los próximos años, plantea todo un paquete de acciones para fomentarlo agrupadas en las siguientes líneas: Sensibilización, Reflexión y Estudio, Apoyo, y Formación y Gestión. Así, su Actuación 2.6 alude a la realización de estudios e investigaciones sobre el concepto, el desarrollo y la importancia del VC en el marco de la RSE3.

De acuerdo con todo lo expuesto, resulta necesario indagar en las razones que pueden estar reteniendo el desarrollo del VC en Europa, y más concreta-

3 Esta debilidad se plantea también en el voluntariado en su conjunto. Tanto es así que el Ministerio de Sanidad, Política Social e Igualdad (2011) identifica en su Reto 3: Mejorar el conocimiento del voluntariado. 
mente en España. La escasa evidencia empírica existente en nuestro país sobre este tema, junto a la influencia de las diferencias culturales inter-países sobre la implementación del VC (Runte et alii, 2010) ${ }^{4}$, apoyan aún más tal necesidad. Este trabajo se propone colaborar en el cierre de esta brecha de desconocimiento y falta de estudios al proponerse como objetivo explorar la veracidad de las razones que puedan estar alentando o retrayendo el desarrollo del VC en nuestro país. Este objetivo se concreta en indagar qué factores pueden estar influyendo positivamente en el hecho de que una empresa practique VC. El resultado orientaría sobre las variables de mayor impacto en la praxis del VC en España a fin de inspirar de forma acertada la toma de decisiones por parte de los organismos y organizaciones competentes en este ámbito. Todo ello con el último propósito de impulsar el desarrollo de estas actividades solidarias desde la perspectiva de la empresa, nuestro sujeto en este análisis.

La selección muestral, con un ámbito temporal del año 2011, se ha realizado conforme a un muestreo subjetivo 0de acuerdo con un criterio razonado, que es el de partir del conjunto de empresas que el Observatorio de VC (2010) considera con mejores prácticas de VC en nuestro país. Tras encuestar a sus directivos responsables de VC a fin de solicitar su percepción acerca de los factores planteados, sometemos los resultados a un análisis descriptivo que nos permite identificar los factores que puedan estar actuando como inhibidores o impulsores del voluntariado en nuestras empresas.

La estructura de este trabajo comienza con una revisión literaria que enmarcará teóricamente el tema y explorará los factores influyentes en el voluntariado de empresa. El resultado de esta revisión se plasma en el diseño de nuestro modelo explicativo de la intensidad con la que se practica VC en España. Será este modelo el que someteremos a contraste empírico por medio de una metodología que, tras la formación muestral basada en las empresas consideradas con mejor VC del país, justifica los indicadores asignados a cada factor explicativo e informa que la obtención de información ha consistido en un análisis de percepciones de los responsables de VC de la muestra recogido por medio de un cuestionario online. Tras analizar los resultados obtenidos, en general favorables al modelo inicial, planteamos las principales conclusiones del trabajo.

4 En este sentido, subrayamos el hecho de que el Eurobarómetro (2008) detectaba que, para la sociedad española, el valor de la solidaridad se encuentra ligeramente superior a la media europea. Así, en 2005 el $12 \%$ de la población española realizaba labores de voluntariado (NBC Spain, 2011b). 


\section{Investigación previa}

\subsection{Marco teórico}

Diversas teorías sirven de soporte conceptual del voluntariado de empresa. Así, la Teoría Institucional (DiMaggio y Powell, 1983) ${ }^{5}$ entiende que las instituciones representan las reglas del juego que las organizaciones están jugando, es decir, estructuran la acción social entre diferentes actores en función de un sistema de incentivos del comportamiento y desempeño de competencias implícito en estructuras de poder y modelos mentales (Vargas, 2005). Esta base teórica nos sirve para deducir que las instituciones no se crean por decreto, sino que son el resultado de un proceso de aprendizaje social; es decir, las organizaciones buscan legitimarse adhiriéndose a los valores deseados por las instituciones clave de la sociedad. En definitiva, la RSE - y más concretamente el VC- surge como una forma de legitimación social de la empresa (Peloza y Hassay, 2006; Basil et alii, 2009) debido a que el VC converge con la misión solidaria que la sociedad comienza a encomendar a las empresas.

La teoría de la organización (Simon, 1962) ofrece una concepción sistémica de la empresa que da soporte al VC al visualizar la empresa como una realidad poliédrica que posee diversas dimensiones y, en consecuencia, debe ser analizada desde diferentes ópticas - incluyendo su entorno-, pero con una visión de conjunto, y también al otorgar al factor humano el rol protagonista de la organización. En suma, esta teoría actúa de soporte del VC al concebir la empresa como una organización, esto es, abierta a la sociedad y con el factor humano en el centro de sus actuaciones.

Por otro lado, la teoría de la agencia (Baiman, 1984), fundamentada en la existencia de información e intereses asimétricos en la empresa, da pie a la lucha por eliminar los costes que esto acarrea; la legitimación de la empresa ante la sociedad en su conjunto - por medio de la RSE - se ofrece como una vía para lograrlo. Así pues, el VC ejerce de reductor de los costes de agencia existentes en la empresa al incrementar la legitimación social de ésta.

También constituye germen del VC, especialmente de su aplicación estratégica en la empresa, la teoría de recursos y capacidades (Penrose, 1959), con las grandes aportaciones posteriores de que las diferencias intrasectoriales debidas a los recursos y capacidades propias de cada empresa generan resultados diferentes entre estas (Rumelt, 1984), y de que estos elementos específicos permiten abordar estrategias con diferentes desempeños (Grant, 1996). La dirección estratégica de Porter (1982) apunta en esta dirección al basar la ventaja competitiva en la

5 No en vano, la teoría institucionalista ha sido aplicada recientemente a la RSE por Campbell (2007). 
generación de capacidades específicas de la empresa a largo plazo - elementos intangibles-. La investigación precedente, como comprobaremos, convierte al VC en un vehículo de generación de potentes capacidades en la empresa: precisamente este cúmulo de intangibles que el voluntariado de empresa genera en ella se encuentra en el centro del presente trabajo.

La teoría de stakeholders (Freeman, 1984), muy vinculada a las anteriores, ofrece un apoyo más específico a la RSE y, con ello, al VC, al orientar la gestión empresarial a la satisfacción voluntaria de los grupos de interés de la empresa. En concreto, el VC permite satisfacer las demandas de stakeholders clave, como son los empleados, la comunidad y, en última instancia, los socios como beneficiarios finales de los efectos de este voluntariado.

Este último enfoque se completa con el modelo conceptual de RSE propuesto por Porter y Kramer (2006), defensor de la integración de los objetivos sociales con las prioridades de la empresa. El núcleo de su modelo se encuentra en el hecho de que diferencia dos grandes visiones en la actuación de la empresa en materia de RSE:

- Receptividad: la empresa realiza actividades sociales y ambientales de índole genérica, o bien destinadas a combatir los efectos negativos producidos por las actividades de su cadena de valor.

- Estratégica: la empresa responde a los problemas sociales y ambientales realizando acciones que mejoran su cadena de valor o su competitividad. Con ello, la RSE se encastra en el análisis estratégico de la empresa. Es en esta perspectiva en la que situamos nuestro trabajo (Porter y Kramer, 2002), esto es, asignamos al VC una naturaleza estratégica para la empresa debido a su inclusión en la RSE.

Esta conjunción de enfoques teóricos permite diseñar un marco conceptual integral para el VC (gráfico 1). 


\section{Gráfico 1: marco teórico del VC}

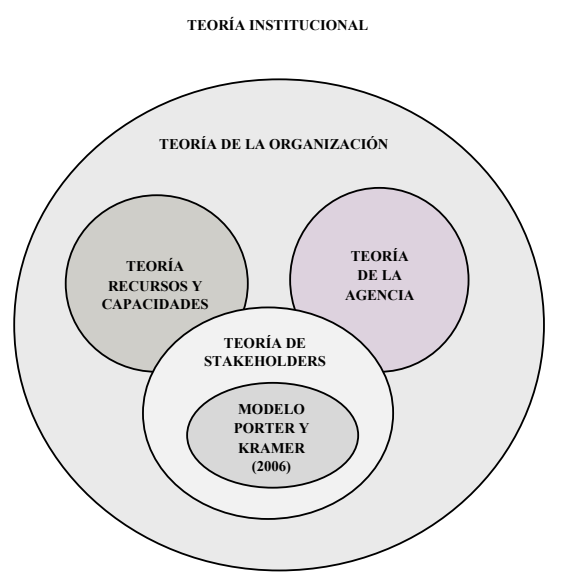

Fuente: elaboración propia.

\subsection{Factores explicativos}

El desarrollo de VC reporta una mejora de la rentabilidad de la empresa (Lewin, 1991; Tuffrey, 2003). En general, la literatura comprueba la existencia de una relación positiva entre la rentabilidad sobre activos de la empresa y su implicación a largo plazo en la sociedad (Waddock y Graves, 1997; Dowell et alii, 2000; Rochlin y Christoffer, 2000) confirmada por el meta-análisis realizado por Orlitzky et alii (2003).

El origen de este incremento de valor añadido para la empresa se sitúa en numerosos factores favorables generados por el VC. Emprendemos aquí la búsqueda estructurada de estos efectos beneficiosos del VC para la empresa. Los estructuramos en factores indirectos del VC (Grupo I), al tratarse de beneficios para la empresa generados por estas prácticas voluntarias $-y$, por tanto, impulsores de ellas- $-y$ aquellos que la investigación previa identifica como de incidencia más directa en las prácticas de voluntariado empresarial (Grupo II). Tras la selección de los factores, en el capítulo de metodología justificaremos y describiremos la forma de medirlos por medio de indicadores.

Grupo I. Factores indirectos del VC (Facilitadores indirectos de las prácticas de VC).

\section{A. Beneficios para la empresa derivados del personal}

En España, León (2010) alude a la conclusión de la Fundación Empresa y Sociedad de que más del $50 \%$ de los trabajadores están a favor de que sus empresas 
colaboren con proyectos sociales. Este atractivo del voluntariado se aprecia mejor entre trabajadores jóvenes; así, es confirmado para EE.UU. por Deloitte (2007), tras detectar que el $62 \%$ de los trabajadores de entre 18 y 26 años prefieren acceder a las empresas que ofrecen programas de voluntariado y que el $97 \%$ de los trabajadores de este intervalo de edad opina que todas las empresas deberían promover este tipo de iniciativas.

Por otro lado, esta práctica de voluntariado revierte en ventajas directas e indirectas para la empresa, estableciéndose así una relación de win-win entre empresa y empleados. Esto conduce a numerosos autores a considerar al VC como parte de la política de recursos humanos (Tuffrey, 2003; León, 2010) en el sentido de que las actividades sociales vinculadas a la empresa mejoran sus recursos humanos (Rochlin y Christoffer, 2000); en concreto, en España las principales variables que relacionan al VC con la política de recursos humanos de la empresa son, y por este orden, la reputación interna, el compromiso para luchar por el logro de los objetivos de la empresa, el clima laboral y la retención de talento (CExS, 2010).

B. Beneficios para la empresa en términos de mejora de su imagen y reputación El compromiso social de la empresa se vincula tanto actualmente tanto su propia reputación que el Índice del Monitor Español de Reputación Corporativa - Merco, (2011) - lo considera una de las variables que lo componen.

En términos generales, De Gilder et alii (2005) muestran que establecer contactos con entidades sin ánimo lucro mejora la imagen de la empresa. No solo el VC representa un recurso intangible eficaz para incrementar la fiabilidad y confianza en la empresa (Muthuri et alii, 2009; Deloitte, 2010) y, por tanto, su reputación (Meijs y Roza, 2010), sino que, si la empresa evita la tentación de realizar acciones de VC para maquillar sus comportamiento de dudosa moral (Miller, 1997), Wild (1993) considera la mejora de la imagen de la empresa que se desprende del VC como el mayor beneficio indirecto de los programas de voluntariado. Esto último está confirmado para diversos países, como Canadá (Basil et alii, 2009): se trata del beneficio del VC más valorado por las empresas de ese país. En nuestro país, este impacto del VC no solo lo confirma CExS (2010) sino que, desde un punto de vista estratégico, una de las dos razones más valoradas por las grandes empresas para desarrollar VC es la de la mejora de la reputación que obtiene la empresa en sí y a través de la implicación de sus empleados en el voluntariado (Observatorio VC, 2010).

Hemos de recordar los efectos tan beneficiosos que la buena reputación genera en la empresa. Entre otros, una consecuencia inmediata de esta mejora es 
la mayor satisfacción de los grupos de interés de la empresa, como los clientes e inversores (Hess et alii, 2002). Concretamente, aumentan las preferencias de los consumidores por los productos de nuestra empresa (Sen y Bhattacharyan, 2001) y atrae a inversores preocupados por destinar sus fondos a inversiones responsables con la sociedad y el medio ambiente (SIF, 1999; Stone, 2001).

\section{Beneficios para la empresa asociados a la mejora de su calidad de gestión}

Caudron (1994) evidencia en EE.UU. que numerosas compañías que llevan a cabo programas de voluntariado perciben que tienen una menor necesidad de confiar algunos servicios a empresas externas debido a su mejor gestión del talento y recursos humanos. Esta afirmación queda contrastada para España cuando $\mathrm{CExS}$ (2010) encuentra que el VC favorece la mejora en la gestión y la eficacia en la toma de decisiones.

\section{Beneficios para los trabajadores que redundan en beneficios indirectos para la empresa}

Las actividades de voluntariado reportan a los empleados intangibles para la gestión de la empresa considerados actualmente de valor estratégico para la dirección de recursos humanos tales son, entre otros, el desarrollo de su creatividad e iniciativa, al tiempo que les permiten mejorar en su conocimiento integral de la empresa (Tuffrey, 1998, 2003; Deloitte, 2010).

\section{E. Beneficios para la empresa debido a que lo son de forma indirecta para la comunidad}

Estamos ante una nueva relación de beneficio recíproco: empresa y comunidad. Esto es así como consecuencia de las valiosas relaciones que establece la primera con la segunda y con las administraciones públicas (Steel, 1995; Guardia et alii, 2006), fruto de las cuales puede aumentar la visibilidad de la empresa en la comunidad (Davidson, 1994). Por otro lado, Points of Light (2000) señala que, por medio del VC, la comunidad recibe muchos beneficios (salud, educación, servicios sociales, ocio, etc.), lo que la convierten en más atractiva y saludable - lo que atrae a más empresas y trabajadores-, al tiempo que beneficia a los trabajadores y sus familias al residir allí (Graff, 2004) — hecho que, a su vez, puede revertir en beneficio de la empresa-. 
Grupo II. Factores directos del VC (Facilitadores directos de las prácticas de $\mathrm{VC})$.

\section{F. Recursos}

Los recursos financieros disponibles en la empresa para atender al personal gestor de los programas de VC, realizar las donaciones a las colaboraciones originadas por el VC, afrontar el diseño y los materiales de la comunicación propia del VC y vincularlos a la cesión del personal de la empresa (Guardia et alii, 2006) condicionan la práctica de VC.

\section{G. Perfil de los trabajadores}

Las características personales y grupales de los empleados de la empresa resultan determinantes sobre el ejercicio de VC por parte la misma en el sentido de que determinadas rasgos de personalidad y de actitud les convierten en propensos a practicar voluntariado, independientemente del apoyo facilitado por la empresa con este fin (Guardia et alii, 2006; Deloitte, 2010, 2011).

\section{H. Perfil de los socios y directivos}

Guardia et alii (2006) consideran que el perfil de los trabajadores de la empresa, así como de los socios y directivos, influyen en su propensión al VC. Como veremos, la edad del trabajador (Peterson, 2004), así como las capacidades que exigen las empresas en una época dada - como sería la actual- (Davidson, 1994), constituyen características del perfil influyentes en la tendencia a involucrarse en actividades de voluntariado.

\section{Estructura organizativa de la empresa}

El tipo de estructura organizativa de la empresa propicia o actúa como barrera del VC en función de si ésta goza de ciertas propiedades que afectan de lleno a la realización de acciones de voluntariado (Guardia et alii, 2006).

\subsection{Modelo}

A partir del conjunto de factores potencialmente explicativos de la realización de actividades de voluntariado por las empresas, hemos creado un modelo que presenta como variable endógena Práctica de VC, es decir, la intensidad con la que la empresa practica el VC (gráfico 2). 
Gráfico 2. Modelo planteado

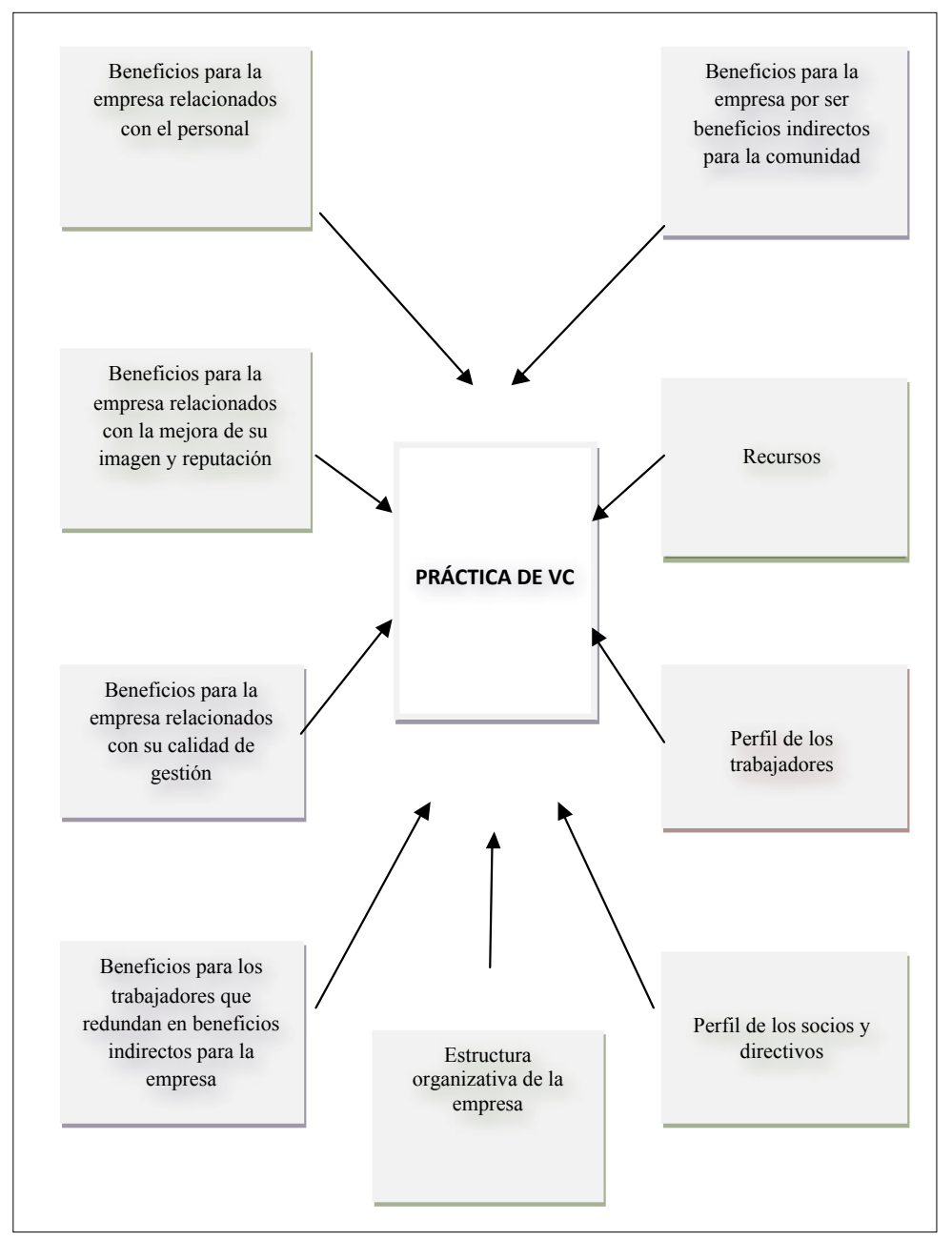

Fuente: elaboración propia.

\section{Metodología}

\subsection{Muestra}

La técnica de selección muestral aplicada ha sido la de muestreo subjetivo aplicando un criterio racional. Concretamente, y de acuerdo con el Informe de VC (2010), partimos del universo muestral formado por las 30 empresas que desarrollan el VC de mejor calidad de nuestro país de acuerdo con el Observatorio de VC (2010) (tabla 1). Elegimos este universo debido a que, en estas empresas, por 
su elevado grado de implicación en el voluntariado, no solo podemos encontrar mejor las claves de la práctica de VC en este país, sino las de un VC de mayor calidad.

Tabla 1. Empresas con mejores prácticas de VC en España

\begin{tabular}{|c|c|c|c|c|c|}
\hline EMPRESA & Sector & EMPRESA & Sector & EMPRESA & Sector \\
\hline ACCENTURE & $\begin{array}{c}\text { Consultora de } \\
\text { negocios }\end{array}$ & DKV & Asegurador & MAPFRE & Asegurador \\
\hline BARCLAYS & Financiero & EROSKI & Distribución & MRW & $\begin{array}{c}\text { Transporte de } \\
\text { Mensajería Urgente }\end{array}$ \\
\hline BAYER & $\begin{array}{l}\text { Químico y } \\
\text { Farmacéutico }\end{array}$ & FERROVIAL & $\begin{array}{c}\text { Gestión de } \\
\text { Infraestructuras y } \\
\text { Servicios } \\
\end{array}$ & NOVARTIS & Salud \\
\hline BBVA & Financiero & $\begin{array}{c}\text { IBERIA } \\
\text { (FUNDACIÓN) }\end{array}$ & $\begin{array}{c}\text { Transporte de } \\
\text { pasajeros }\end{array}$ & SANTANDER & Financiero \\
\hline BT & $\begin{array}{c}\text { Comunicación y } \\
\text { Tecnología }\end{array}$ & $\begin{array}{c}\text { REPSOL } \\
\text { (FUNDACIÓN) }\end{array}$ & Energético & SEUR & Logística Integral \\
\hline BANKIA & Financiero & GRUPO VIPS & $\begin{array}{l}\text { Hostelería y } \\
\text { comercio }\end{array}$ & TELEFÓNICA & Telecomunicaciones \\
\hline CEPSA & Energético & IBERDROLA & Energético & TIMBERLAND & Textil \\
\hline CITI & Financiero & IBM IBÉRICA & $\begin{array}{c}\text { Tecnologías de la } \\
\text { Información y la } \\
\text { Comunicación }\end{array}$ & UNILEVER & Gran Consumo \\
\hline CONSUM & Gran consumo & CAIXA & Financiero & $\begin{array}{c}\text { URÍA \& } \\
\text { MENÉNDEZ }\end{array}$ & Abogacía \\
\hline DISNEY & Audiovisual & LILLY & $\begin{array}{c}\text { Químico y } \\
\text { Farmacéutico }\end{array}$ & $\begin{array}{c}\text { USP } \\
\text { HOSPITALES }\end{array}$ & $\begin{array}{c}\text { Gestión de } \\
\text { instituciones } \\
\text { sanitarias }\end{array}$ \\
\hline
\end{tabular}

La formación muestral se ha realizado conforme a la información recogida en la tabla 2 .

\section{Tabla 2. Ficha técnica del trabajo de campo}

\begin{tabular}{|l|l|}
\hline Universo & $\begin{array}{l}\text { Empresas con mejores prácticas } \\
\text { de VC que operan en España } \\
\text { (Observatorio de VC, 2010) }\end{array}$ \\
\hline Ámbito espacial & España \\
\hline Período muestral & 2011 \\
\hline Forma de contacto & $\begin{array}{l}\text { Personal telefónico para presentar } \\
\text { el trabajo, solicitar participación y } \\
\text { comunicar envío de encuestas }\end{array}$ \\
\hline Método de captación de información & Encuesta electrónica \\
\hline Unidad muestral & $\begin{array}{l}\text { Persona encargada de la RSC/VC } \\
\text { en cada empresa }\end{array}$ \\
\hline Universo muestral & 30 \\
\hline Muestra final & 18 \\
\hline Índice de respuesta (\%) & $60 \%$ \\
\hline &
\end{tabular}

Fuente: elaboración propia. 
Atendiendo a su número de empleados (superior a 250), la muestra está formada por grandes empresas. Los resultados, por tanto, pese a que se podrían aplicar a pymes con la debida cautela y limitaciones, y como orientación para ellas, identifican las variables que inciden en el VC para este colectivo de empresas.

Así pues, determinaremos los factores que mueven a las grandes empresas a practicar un VC de excelencia en España.

\subsection{Medición de las variables}

La naturaleza cualitativa de estas variables nos aconseja aplicar un sistema de escala de medida para cada una de ellas. Se trata de identificar los indicadores que podrían medir cada uno de estos factores. Debido a la inexistencia en la literatura de una escala de medida aplicable para cada factor considerado que se plantee con los objetivos de este trabajo, procedemos a diseñarla de forma justificada. Los indicadores resultantes se muestran en la tabla 3.

\section{Factor A: Beneficios para la empresa relacionados con el personal}

A1. Existe un vínculo entre VC y el desarrollo y mejora de las habilidades y capacidades de los empleados que les permiten afrontar los retos relacionados con su trabajo (Caudron, 1994, Finney, 1997, Hoare, 2004). Esta virtud es aún más valorada por las empresas actuales al requerir un perfil profesional muy volcado en las aptitudes y actitudes de los empleados; esta razón convierte a este voluntariado en un atractivo para que los trabajadores más jóvenes se sumen a él (Peterson, 2004a).

Entre las habilidades que desarrolla y mejora la calidad del trabajo se encuentra la de liderazgo, muy valorada por los trabajadores actuales dada su demanda de promoción en la empresa (Davidson, 1994); por esta razón, cabría plantearse el VC como herramienta de formación interna con esta finalidad. El trabajo en equipo (Peloza y Hassay, 2006), la comunicación, la gestión de proyectos y la generación de contactos con utilidad para el trabajo (Peterson, 2003), o la colaboración, son otras habilidades que se ejercitan con el VC. En realidad, casi todas las habilidades pueden ser desarrolladas con una gestión del VC orientada a este propósito, es decir, planificando, organizando, ejecutando y controlando los resultados del voluntariado aplicado (Tuffrey, 1998). Estas adquisiciones o mejoras, junto con la mayor autoestima que le producen, permiten al trabajador aumentar su capacidad para resolver problemas proactivos en el trabajo (Geroy et alii, 2000).

En España también se puede contemplar al VC como herramienta de desarrollo profesional, ya que se considera de manera mayoritaria que fortalece el 
liderazgo, la adaptabilidad, la comunicación, el trabajo en equipo, la gestión de proyectos y, en general, competencias personales y profesionales que enriquecen el perfil profesional del empleado (CExS, 2010). De este modo, este Factor A resulta ser el más valorado por las grandes empresas españolas tras detectar el Observatorio de VC $(2009,2010)$ que los principales objetivos que persiguen las empresas a la hora de desarrollar voluntariado, desde un punto de vista operativo, son fomentar el orgullo de pertenencia a la empresa, mejorar el clima de la organización, la captación y retención del talento, así como el desarrollo de los empleados.

A2. Las actividades de voluntariado refuerzan el sentido de pertenencia a la empresa por parte del trabajador (Carroll, 1990). La mayoría de los empleados, a igualdad de condiciones, elegirían una empresa con VC; como resultado, la gran mayoría de los empleados de empresas con VC no solo se sienten orgullosos de trabajar en ella (Deloitte, 2011), sino que se encuentran más satisfechos de trabajar aquí que en otras empresas (Tuffrey, 2003). Esta razón les lleva a recomendar a sus amigos trabajar en ella. En definitiva, estas labores voluntarias identifican al trabajador con la empresa y les hace sentir orgullo de pertenecer a la misma (CExS, 2010).

Igualmente, el voluntario de empresa en España declara que, además de motivarle hacia el VC valores personales relacionados con la solidaridad, este voluntariado significa para ellos una oportunidad de aprender nuevas experiencias y de poner en práctica conocimientos y habilidades que únicamente se adquieren así (Dávila, 2010). En este país, la implicación de la empresa en la sociedad se sitúa como el cuarto factor que inspira el orgullo de un trabajador de pertenecer a su empresa - tras las posibilidades de desarrollo profesional, la reputación de la empresa y la calidad de sus productos y servicios, por ese orden- (CExS, 2010).

A3. Consecuencia de muchos de los efectos favorables que produce en el trabajador, las acciones de voluntariado permiten mejorar el clima laboral (Wild, 1993), algo también confirmado en España (CExS, 2010).

A4. La mejora en las habilidades que posibilita el VC permite atraer a nuevo capital humano a la empresa. Esto se observa aún en mayor medida para trabajadores jóvenes (Deloitte, 2007; Peterson, 2004a), a fin de trasladar a la sociedad sus habilidades profesionales, y altamente cualificados (Wild, 1993; Caudron, 1994; Backhaus et alii, 2002); los empleados de mayor edad se ven atraídos por el enriquecimiento de relaciones sociales que genera el VC (Peterson, 2004a). Recuperamos, así mismo, los argumentos citados con anterioridad acerca de la elección mayoritaria de una empresa con VC y la recomendación de trabajar en 
ella que desvelaba Deloitte (2011). En suma, la mayoría de las empresas con buenos programas de VC atraen de forma consistente a los mejores nuevos talentos (Rochlin y Christoffer, 2000) debido a que los empleados están demostrando cada vez con más fuerza que desean trabajar para una empresa que muestre un buen comportamiento con la ciudadanía (Pajo y Lee, 2011).

Esto conduce a que la mayoría de las empresas con VC están intentando utilizarlo para atraer a nuevos trabajadores, lo que reduce los costes de contratación y de formación de la empresa (Points of Light, 2000).

A5. Ya Carroll (1990) hallaba evidencia de que el VC reforzaba el sentimiento de lealtad del trabajador con la empresa, un hecho que se ha prolongado en el tiempo - especialmente con empleados de alta cualificación- (Wild, 1993; Caudron, 1994; Backhaus et alii, 2002), y que continúa hasta nuestros días al comprobar Deloitte (2011) que la gran mayoría de los empleados se sienten más leales con la empresa con VC, incluida España (CExS, 2010). En consecuencia, se observan mayores tasas de retención de empleados en las empresas que aplican VC (Tuffrey, 2003).

Así pues, el atractivo del VC provoca que se esté intentando convertirlo en herramienta para retener trabajadores (Points of Light, 2000), de nuevo con el consiguiente ahorro de costes de contratación y formación.

A6. El voluntariado mejora la satisfacción de los empleados en el trabajo (Stebbins, 1989; De Gilder et alii, 2005); tanto es así que la gran mayoría de los que trabajan en empresas con VC se sienten muy satisfechos en él (Deloitte, 2011), de igual modo en España (CExS, 2010). Aún más, los trabajadores que practican voluntariado gozan de unos mayores niveles de satisfacción (Tuffrey, 2003).

A7. El VC aumenta el grado de compromiso del trabajador con la empresa (Carroll, 1990; De Gilder et alii, 2005). En términos comparados, Peterson (2004b) encuentra un mayor compromiso del trabajador con la empresa en las que aplican actividades de voluntariado que en las restantes.

Factor B: Beneficios para la empresa relacionados con la mejora de su imagen y reputación

La reputación de una empresa es una variable multidimensional y compleja de definir y medir (Suviri, 2010). Para medir este factor seguimos al Foro de Reputación Corporativa (2012) - representante en España de Reputation Institute-. Éste mide la reputación a través de una metodología denominada RepTrack y siete dimensiones: Oferta, Trabajo, Finanzas, Liderazgo, Innovación, Ciudadanía y Gobierno, a cada una de las cuales se le asigna un conjunto de in- 
dicadores. Dados los objetivos de nuestro trabajo y por razones de operatividad, de ellos hemos elaborado uno para cada dimensión ${ }^{6}$. Se trata de los indicadores de naturaleza perceptiva.

Factor C: Beneficios para la empresa relacionados con la mejora de su calidad de gestión

Montañés y Olier (2006) ofrecen una noción de calidad de la gestión de una empresa basada en su gestión de la diversidad, es decir, consistente en la creación de valor para los stakeholders implicados en la empresa. Concretamente, fusionan la disciplina de la dirección estratégica con la basada en la dirección de los sistemas de información para crear una nueva misión para todas las organizaciones. Elegimos este enfoque dados los objetivos y metodología de nuestro trabajo, el cual, aplicado a los grupos de interés de una empresa considerados por AECA (2004), nos inspiran los indicadores que medirán este factor.

Factor D: Beneficios para los trabajadores que redundan en beneficios indirectos para la empresa

D1. Cuadron (1994) encuentra evidencias de que las actividades de voluntariado fomentan la creatividad y el espíritu de iniciativa de los empleados. Tuffrey (1998) ordena sus resultados y publica que el pensamiento creativo resulta ser la tercera habilidad con incidencia profesional que desarrolla el voluntario de empresa (tras la comunicación y la colaboración y trabajo en equipo). Años más tarde, CExS (2010) reafirma este indicador para España.

D2. La práctica de VC eleva la moral y autoestima de los empleados de la empresa (Lewin, 1991; Tuffrey, 2003; Peloza y Hassay, 2006; Basil et alii, 2009; Deloitte, 2010; CExS, 2010).

D3. Los empleados voluntarios ganan en interés por sus jefes, sus colegas y los de otros departamentos de la empresa, aumentando su relación personal con ellos, al tiempo que ganan en conciencia de que todos luchan por un objetivo común (Tuffrey, 2003; Meijs y Roza, 2010). En definitiva, estos mayores contactos y relaciones con el resto de la empresa les hace mejorar la visión integral de la empresa (Guardia et alii, 2006; CExs, 2010).

6 Algunos resultan de la unión de varios de la dimensión correspondiente. 
Factor Eः Beneficios para la empresa por ser beneficios indirectos para la comunidad

E1. La comunidad recibe vía voluntariado empresarial incuestionables beneficios - salud, educación, servicios sociales, ocio, etc. — que permiten generar un entorno propenso a congeniar con la empresa (Steel, 1995; Wild, 1993; Basil et alii, 2009; Points of Light, 2000). Esta realidad deriva en:

E2. Una mejora de las relaciones de la empresa con la comunidad (Cone/ Roper, 1999; Maignan et alii, 1999; Points of Light, 2000; CExS, 2010).

E3. Unas relaciones más fluidas de la empresa con las administraciones públicas (CExS, 2010).

\section{Factor F: Recursos}

De acuerdo con el Observatorio del Tercer Sector (2007), la disponibilidad de recursos financieros por parte de la empresa actúa como facilitador de la puesta en marcha de adecuado VC. Los fondos precisos para ello están relacionados con los diversos ámbitos de actuación del voluntariado:

F1. Los costes, en general, y el coste de personal en particular. La indisponibilidad de fondos suficientes para atenderlos se convierte en barrera para la aplicación del VC (Basil et alii, 2009).

F2. Si la empresa decide no solo promover el VC, sino cooperar directamente con él en forma de contribuciones económicas y en especie, resulta obvia la necesidad de disponer de fondos para ello.

F3. Deben financiarse el diseño y la adquisición de materiales de comunicación. Points of Light (1999) evidencia que los costes administrativos actúan de barrera para el VC.

F4. Deloitte (2011) detecta que la mayoría de los trabajadores que no se suman al voluntariado es a causa de que no disponen de tiempo para ello, lo que encierra una falta de recursos de la empresa para ofrecer esta forma de voluntariado en especie.

\section{Factor G: Perfil de los trabajadores}

Guardia et alii (2006), tras apuntar al perfil de los trabajadores de la empresa como una de las variables explicativas del VC realizado, añaden que la aspiración de los trabajadores a progresar dentro de la empresa, su grado de implicación 
en el entorno y de compromiso con la empresa y la duración de los contratos de trabajo ejercen poder sobre la decisión de implantar VC.

El indicador de expectativas de los trabajadores a promocionar dentro de la empresa es apoyado por Deloitte (2011). La tendencia de los empleados a implicarse en los problemas del entorno condiciona el voluntariado de la empresa, dado que, por un lado, los trabajadores se sienten implicados en su entorno (Deloitte, 2011), y por otro, lo consideran una forma eficaz de colaborar - al hacerlo la empresa con entidades sin ánimo de lucro- para aliviar los problemas sociales (Deloitte, 2010).

\section{Factor H: Perfil de los socios y directivos}

La influencia de los socios y directivos en las prácticas de voluntariado se concreta en la apuesta de los directivos por la solidaridad social de la empresa y sus empleados, así como en el grado de implicación real de la alta dirección y de alineación de los directivos de los restantes niveles con la alta dirección de la empresa (Guardia et alii, 2006).

En este sentido, Peloza y Hassay (2006) encuentran que el apoyo de la dirección (a todos sus niveles) impulsa al voluntariado a aquellos trabajadores que buscan reconocimiento u otras recompensas. Deloitte (2010) demuestra cómo el VC llega a conseguir el interés personal de la Alta Dirección de la empresa.

\section{Factor I: Estructura organizativa de la empresa}

Las propiedades de la estructura organizativa de la empresa propicia o entorpece el VC en el sentido de que favorecerá la implementación de actividades programadas de VC si la estructura se adapta fácilmente a la evolución del entorno, los directivos delegan en sus subordinados, la comunicación interna fluye sin dificultad y la empresa dispone de personal dedicado a gestionar el VC (Guardia et alii, 2006). Pese a que la mayoría de directivos consideran que las actividades de VC encajan bien dentro de su organización (Meijs y Roza, 2010), el Observatorio del Tercer Sector (2007) desgrana la relevancia de esta variable e incluso sostiene la necesidad de cambiar la estructura organizativa si es preciso para facilitar los programas de voluntariado.

\section{Factor J: Prácticas de voluntariado corporativo en la empresa}

Estamos ante nuestra variable objetivo o endógena. A la luz de nuestros objetivos, así como de los factores y sus indicadores atribuidos, esta variable, como expresión del grado de implicación de la empresa en actividades de VC, resultará medida por los atributos referidos en la tabla 3. 
Tabla 3. Factores explicativos e indicadores

\begin{tabular}{|c|c|c|c|}
\hline Factor & Indicadores & Factor & Indicadores \\
\hline $\begin{array}{c}\text { A } \\
\text { Beneficios para } \\
\text { la empresa } \\
\text { relacionados } \\
\text { con el personal }\end{array}$ & $\begin{array}{l}\text { A1. Desarrollo de competencias y } \\
\text { habilidades de los empleados } \\
\text { A2. Sentido de pertenencia a la empresa } \\
\text { del trabajador } \\
\text { A3. Mejorar el clima laboral } \\
\text { A4. Atraer a nuevo capital humano a la } \\
\text { empresa } \\
\text { A5. Incremento de la lealtad de los } \\
\text { trabajadores } \\
\text { A6. Incremento de la satisfacción de los } \\
\text { empleados en el trabajo } \\
\text { A7. Favorecer o fortalecer el compromiso } \\
\text { del trabajador con la empresa }\end{array}$ & $\begin{array}{c}\mathrm{F} \\
\text { Recursos }\end{array}$ & $\begin{array}{l}\text { F1. Financiación del coste del personal } \\
\text { de la empresa que gestione los programas } \\
\text { de VC } \\
\text { F2. Recursos financieros para realizar } \\
\text { contribuciones económicas y en especie a } \\
\text { las colaboraciones del VC } \\
\text { F3. Financiación para el diseño y } \\
\text { materiales de comunicación implicados } \\
\text { en VC } \\
\text { F4. Financiación disponible para la cesión } \\
\text { de personal especializado de la empresa }\end{array}$ \\
\hline $\begin{array}{l}\text { B } \\
\text { Beneficios para } \\
\text { la empresa } \\
\text { relacionados } \\
\text { con la mejora } \\
\text { de su imagen y } \\
\text { reputación }\end{array}$ & $\begin{array}{l}\text { B1. Percepción de los trabajadores de su } \\
\text { justa consideración por la empresa } \\
\text { B2. Percepción por parte del cliente de que } \\
\text { la empresa está orientada a su satisfacción }{ }^{1} \\
\text { B3. Percepción de los socios de que } \\
\text { la empresa obtiene buenos resultados } \\
\text { financieros } \\
\text { B4. Percepción de la gestión de la empresa } \\
\text { como de calidad } \\
\text { B5. Considerar a la empresa como } \\
\text { innovadora } \\
\text { B6. Consideración de la empresa como } \\
\text { responsable con la sociedad y el medio } \\
\text { ambiente } \\
\text { B7. Consideración de la empresa como no } \\
\text { abusiva con el poder que tiene }\end{array}$ & $\begin{array}{c}\mathrm{G} \\
\text { Perfil de los } \\
\text { trabajadores }\end{array}$ & $\begin{array}{l}\text { G1. Los trabajadores aspiran a progresar } \\
\text { dentro de la empresa } \\
\text { G2. Los trabajadores se sienten } \\
\text { implicados en su entorno } \\
\text { G3. Los trabajadores están } \\
\text { comprometidos con la empresa } \\
\text { G4. Los trabajadores de la plantilla lo son } \\
\text { mediante contratos temporales }\end{array}$ \\
\hline $\begin{array}{c}\text { C } \\
\text { Beneficios para } \\
\text { la empresa } \\
\text { relacionados } \\
\text { con la mejora } \\
\text { de su calidad de } \\
\text { gestión }\end{array}$ & $\begin{array}{l}\text { C1. Satisfacción de las expectativas de los } \\
\text { socios por la empresa } \\
\text { C2. Satisfacción de las expectativas de los } \\
\text { empleados por la empresa } \\
\text { C3. Satisfacción de las expectativas de los } \\
\text { clientes por la empresa } \\
\text { C4. Satisfacción de las expectativas de los } \\
\text { proveedores por la empresa } \\
\text { C5. Satisfacción de las expectativas de las } \\
\text { administraciones públicas por la empresa } \\
\text { C6. Satisfacción de las expectativas de la } \\
\text { comunidad local por la empresa } \\
\text { C7. Satisfacción de las expectativas de la } \\
\text { sociedad en general por la empresa }\end{array}$ & $\begin{array}{c}\mathrm{H} \\
\text { Perfil de } \\
\text { los socios y } \\
\text { directivos }\end{array}$ & $\begin{array}{l}\text { H1. Los socios apuestan por la } \\
\text { solidaridad social de la empresa y sus } \\
\text { empleados } \\
\text { H2. La Alta Dirección de la empresa } \\
\text { defiende y aplica con convencimiento } \\
\text { personal la implicación social de la } \\
\text { empresa y sus } \\
\text { empleados } \\
\text { H3. Los directivos de no Alta Dirección } \\
\text { comparten con la Alta Dirección el } \\
\text { convencimiento personal del compromiso } \\
\text { social de la empresa y sus empleados, y } \\
\text { la apoyan }\end{array}$ \\
\hline $\begin{array}{l}\text { D } \\
\text { Beneficios para } \\
\text { los trabajadores } \\
\text { que redundan } \\
\text { en beneficios } \\
\text { indirectos para } \\
\text { la empresa }\end{array}$ & $\begin{array}{l}\text { D1. Mejorar la creatividad y el espíritu de } \\
\text { iniciativa de sus empleados } \\
\text { D2. Elevar la moral y autoestima de sus } \\
\text { empleados } \\
\text { D3. Visualización de la empresa de forma } \\
\text { integral por los trabajadores }\end{array}$ & $\begin{array}{c}\text { I } \\
\text { Estructura } \\
\text { organizativa } \\
\text { de la empresa }\end{array}$ & $\begin{array}{l}\text { I1. La empresa presenta una estructura } \\
\text { de recursos humanos que se adapta } \\
\text { fácilmente a los cambios del entorno } \\
\text { I2. Existe delegación de los directivos en } \\
\text { otros jerárquicamente inferiores } \\
\text { I3. Se da una comunicación interna fluida } \\
\text { en la empresa } \\
\text { I4. La empresa dispone de personal } \\
\text { dedicado a gestionar el VC }\end{array}$ \\
\hline $\begin{array}{l}E \\
\text { Beneficios para } \\
\text { la empresa por } \\
\text { ser beneficios } \\
\text { indirectos para } \\
\text { la comunidad }\end{array}$ & $\begin{array}{l}\text { E1. Creación de un entorno estable y } \\
\text { saludable de la comunidad donde la } \\
\text { empresa opera } \\
\text { E2. Mejorar las relaciones de la empresa } \\
\text { con la comunidad } \\
\text { E3. Mejorar las relaciones de la empresa } \\
\text { con las administraciones públicas }\end{array}$ & $\begin{array}{c}\text { J } \\
\text { Prácticas de } \\
\text { voluntariado } \\
\text { corporativo en } \\
\text { la empresa }\end{array}$ & $\begin{array}{l}\text { J1. La empresa promueve y apoya al } \\
\text { voluntario empleado para que colabore } \\
\text { con la sociedad y/o el medio ambiente } \\
\text { J2. La empresa dispone de diversos } \\
\text { programas de VC } \\
\text { J3. El personal y directivos de la empresa } \\
\text { participan en los programas de VC } \\
\text { J4. La empresa dedica recursos, } \\
\text { diferentes al personal y directivos, en su } \\
\text { participación en los programas de VC }\end{array}$ \\
\hline
\end{tabular}


1 Apoyado por Points of Light (2000) para el VC.

2 Se confirma expresamente para el VC tras comprobarse que este voluntariado impulsa la innovación (Fundación Seres, 2011), especialmente en productos y mercados (Rochlin y Christoffer, 2000). Precisamente una de las dos principales razones estratégicas de las grandes empresas españolas para realizar VC está relacionada con las oportunidades de negocio que genera a consecuencia de la innovación que conllevan estas actividades solidarias (Observatorio de VC, 2010).

3 Basil y Erlandson (2008) y Rochlin y Christoffer (2000) demuestran que el VC mejora las relaciones con los clientes. Incluso está en mente de los mismos trabajadores, dado que la mayoría de los encuestados por Deloitte (2010) consideran que con VC ayudan más a los clientes.

4 Smith (2003) y Den Hond et alii (2007) lo confirman explícitamente para el VC.

5 Hallamos evidencia de este efecto del VC en Tuffrey (1998), Points of Light (2000), Smith (2003) y Den Hond et alii (2007).

6 Para los dos últimos indicadores (C7 y C8), cabe mencionar a Tuffrey (1998), Smith (2003) y Den Hond et alii (2007) como investigaciones que confirman este impacto del VC.

7 No resulta suficiente con promover las relaciones entre empleados, aunque sí necesario (Peloza y Hassay, 2006).

\subsection{Obtención de información}

Nuestro objetivo aconseja plantear nuestros interrogantes como un análisis de percepciones. La naturaleza cualitativa de los factores planteados como determinantes de la práctica de VC en España obliga a la identificación de los indicadores que las midan, es decir, al diseño de una escala de medida. Para esto último, hemos asignado a cada indicador una escala de Likert de 0 a 10 , donde 0 significa otorgarle a la cuestión ningún valor y 10 la máxima valoración (Rodríguez y García, 2011). Lewis y Mackenzie (2000) la aplican para estudiar si los inversores éticos británicos están dispuestos a invertir en empresas socialmente irresponsables.

Hemos optado por un modelo de encuesta online por las evidentes ventajas que reporta. Para minimizar el riesgo de no ser respondido por el responsable previsto, contactamos previamente con él a fin de persuadirle de que nos los contestara personalmente, dado el interés del trabajo y, en particular, de este sondeo que contiene ${ }^{7}$.

El cuestionario se encuentra dividido en dos partes básicas: Identificación de la empresa y Factores del VC: <https://docs.google.com/spreadsheet/viewfor $\mathrm{m} ? \mathrm{hl}=\mathrm{es} \&$ formkey $=\mathrm{dDRTcVNKMTRmVlV5VFdqdGVON0hQRXc6MQ}$ \#gid $=0>$

El tratamiento de las respuestas se ha realizado con el programa SPSS versión 18.

7 Agradecemos en este sentido la colaboración tan directamente recibida por parte de los directivos responsables de las firmas que componen la muestra. 


\section{Análisis de resultados}

Procedemos al análisis descriptivo de los resultados obtenidos para cada factor y sus indicadores tras ordenar estos últimos de acuerdo con su valoración media recibida (tabla 4).

\section{FACtor A}

Con este Factor A, preguntamos a las empresas sobre los beneficios que obtienen de su personal por llevar a cabo políticas de VC. Si observamos la tabla 4 y el gráfico 3 (Anexo), comprobamos cómo los indicadores se ven fuertemente respaldados: se sitúan en la franja alta del notable — a excepción de A4-, lo que confirma que las empresas valoran en gran medida las ventajas que ofrece el VC a sus empleados — vinculadas con el desempeño empresarial— a la hora de decidir si aplicar voluntariado.

Destaca especialmente A2 (orgullo de pertenencia), seguido de A6 (satisfacción de los empleados) y, muy próximo, el fortalecimiento del compromiso del trabajador con la empresa (A7), es decir, beneficios del voluntariado asociados directamente al bienestar personal de los empleados y al vínculo emocional con la empresa que se desprende de esto.

Por coherencia con este resultado, la mejora de competencias y habilidades de los empleados - VC como herramienta directa de desarrollo profesional- (A1) se sitúa solo en cuarto lugar de preferencia. Estos resultados se alinean con los resultados del Observatorio de VC $(2009,2010)$, recordemos, que colocaba al orgullo de pertenencia como el objetivo de VC más considerado por las grandes empresas en España y valoraba A1 en menor medida.

Destacamos también que el VC como mecanismo de retención del talento (A4) se descuelga del notable, pese a su incontestable aprobado: recordando a Runte et alii (2010), posiblemente influyan nuestras diferencias culturales respecto de otros países que puedan valorarlo más (tales como EE.UU.). 


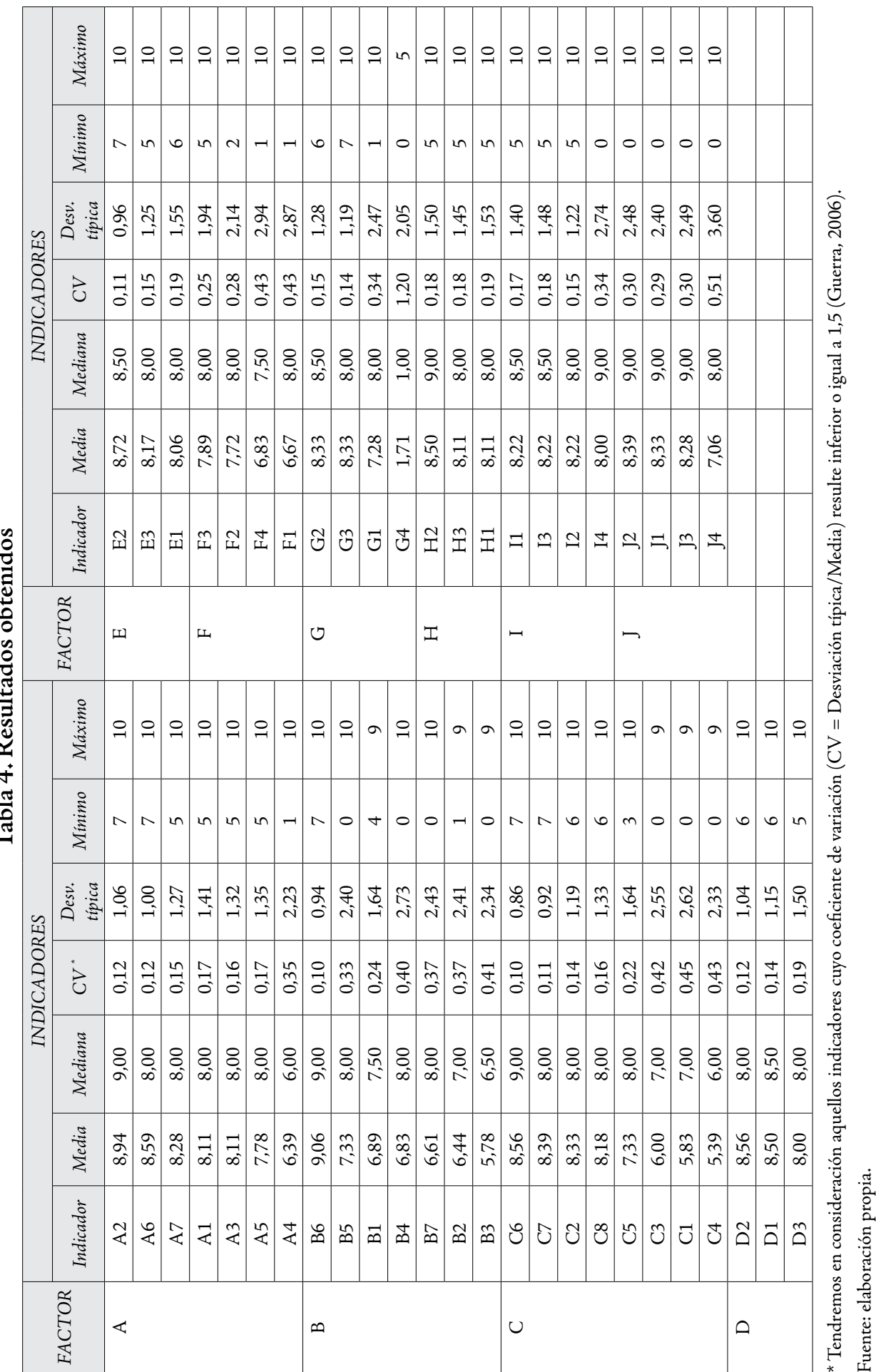




\section{Factor B}

Para este Factor B (tabla 4, gráfico 4 -Anexo-), nos encontramos con resultados dispares, pero todos tienen en común que alcanzan un valor medio de aprobado: la empresa con VC percibe una mejora de su imagen y reputación a resultas del mismo.

Destaca sobremanera el indicador que manifiesta su comportamiento de compromiso ecosocial (B6), lo que indica que, fundamentalmente, la empresa con VC valora la mejora de reputación que obtiene a través de la imagen de empresa con RSE que ofrece — resultado coherente con los del Factor A- A cierta distancia, pero a un nivel notable, la empresa valora que sea considerada como innovadora por el hecho de practicar voluntariado (B5).

En una tercera franja se sitúan las percepciones de los trabajadores y clientes (B1, B2), pero no tanto la de mejora de resultados financieros por parte de los socios (B3): resulta aprobada, pero con nota muy discreta, la influencia de los beneficios empresariales sobre la decisión de la empresa de practicar voluntariado; es decir, pesa más sobre la decisión la mejora de la gestión de la empresa que genera el VC que el aumento de beneficios que produce.

\section{FACtor C}

Los resultados (tabla 4, bráfico 5-Anexo-) sugieren que las empresas de la muestra se ven influidas por las mejoras que produce el VC en la calidad de su gestión a la hora de decidirse o no por el voluntariado.

Pero los resultados segmentan estas razones en tres grupos: destacan - con notable alto-, y en coherencia con los resultados de los factores A y B, el logro de objetivos vinculados al cumplimiento de las expectativas de sus empleados $y$ de la comunidad/sociedad (C2, C6, C7, C8). Una franja de interés intermedio la representa el atender las expectativas de clientes y administraciones públicas $(\mathrm{C} 3$, C5), grupos de interés por tanto menos considerados en su decisión de aplicar VC; sorprende que las administraciones públicas se sitúen por encima de los clientes, quizá indicativo del fomento de la RSE por parte de los poderes públicos en el país (dirigidos por la UE) en los últimos años, y que tal vez encierre también un deseo de buenas relaciones con las instituciones públicas por medio del voluntariado. Finalmente, las razones menos aplaudidas son las relacionadas con los socios - de nuevo-y proveedores (C1, C4).

\section{FACTOR D}

Las empresas no solo admiten todos estos indicadores como razones para implementar voluntariado, sino que el peso que alcanzan es elevado (tabla 4, gráfico 
6 -Anexo-). Junto a los resultados del Factor A, esto confirma que los motivos asociados a los empleados se sitúan en un lugar preferente a la hora de decidirse por el VC.

Además, estos resultados del Factor $\mathrm{C}$ se alinean con los obtenidos para el Factor $\mathrm{A}$ en el sentido de que el orgullo de pertenencia a la empresa, la satisfacción en el trabajo y el aumento de moral y autoestima que logra el trabajador se convierten en los tres principales argumentos relacionados con los empleados por los que la empresa acepta realizar VC. Es decir, razones de satisfacción del empleado y, por ende, satisfacción de éste con su empresa. No obstante, se sitúan bastante próximos los efectos que esta satisfacción podrían estar originando en la empresa (tales como A3, A5, A7), así como el desarrollo de competencias y habilidades que consigue el trabajador voluntario (A1).

\section{FaCtor E}

Las empresas declaran que las mejoras que produce el VC en su entorno y en las relaciones con él que de esto se deriva se convierten en un objetivo de sus actividades de voluntariado; un objetivo, además, muy valorado por las empresas al alcanzar un notable alto (tabla 4, gráfico 7 -Anexo-).

Destaca la mejora de sus relaciones con la comunidad (E2) por encima de las que mantiene con las administraciones públicas (E3). En relación con las segundas, los resultados parecen indicar el mayor interés de las empresas por unas relaciones fluidas con los poderes públicos (E3) que por la satisfacción de las expectativas de estos (C5) como causas que les impulsen a ejercer VC.

\section{FACTOR F}

La tabla 4 (gráfico 8-Anexo-) nos revela que la disponibilidad de los recursos financieros en las empresas condiciona la puesta en marcha de actividades de voluntariado. Pese a ello, su influencia se percibe como algo menor que factores más potentes como los relacionados con los trabajadores y el entorno (comunidad, administraciones públicas, sociedad).

Destacan la financiación precisa para el sistema de comunicación con los empleados (F3) y, obviamente, para afrontar las contribuciones directas de la empresa a los destinatarios de su VC (F2). Menos afecta el coste de la cesión de personal especializado para labores de voluntariado - que no alcanza siquiera el notable- (F4), un resultado de interés, y el coste del personal vinculado a la gestión de los programas de voluntariado (F1). 


\section{FACTOR G}

Los resultados sugieren que el perfil de los trabajadores de la empresa afecta a la decisión de la misma sobre si practicar o no VC (tabla 4, gráfico 9 -Anexo-) $y$, además, lo hace con una intensidad notable. Tan solo el carácter temporal o indefinido de los contratos de la plantilla resulta indiferente (G4), lo que quizá puede encerrar que la disposición del trabajador temporal al voluntariado es equiparable a la del trabajador con contrato indefinido.

Especialmente influyen en la decisión de la empresa el grado de implicación de los empleados con su entorno (G2) y, en igual medida, el nivel de compromiso de los empleados con la empresa (G3); es decir, las empresas necesitan partir de una plantila solidaria, un requisito necesario pero no suficiente dado que, además, debe sentirse parte de la empresa - lo que puede estar relacionado con la labor de motor del voluntariado que esta última ejerce y con la calidad del voluntariado que exige-.

Observamos cómo las empresas tienen muy en consideración, aunque algo menos, la demanda de promoción en la empresa de sus trabajadores, lo que puede estar en sintonía con las altas puntuaciones asignadas a los indicadores de factores anteriores que recogían beneficios para los trabajadores vinculados a su progresión profesional (tales como A1, D1, D2, D3).

\section{Factor H}

Los resultados del Factor $\mathrm{H}$ ( tabla 4, gráfico 10 - Anexo-) sugieren que el nivel de implicación social de los socios y directivos de la empresa influye en la toma de decisión de desarrollar actividades de voluntariado; incluso se convierte en una variable potente en esta toma de decisión — todos los ítems con notable-.

Destaca el peso de la Alta Dirección (H2) — por encima incluso de los socios-, lo que confirma resultados de trabajos como el Observatorio de VC (2011) cuando señala que la implicación de la Alta Dirección —así como de los mandos intermedios - de la empresa resulta clave para el éxito de los programas de voluntariado ${ }^{8}$. Esta valoración tan elevada de la Alta Dirección apoya la tesis de que el VC que se esté aplicando sea de carácter estratégico (Basil et alii, 2009, Fundación Seres, 2011).

\section{FACTOR I}

Los resultados de este Factor I (tabla 4, gráfico 11 -Anexo-) destacan la relevancia de la estructura organizativa en el VC y, por tanto, en la decisión de

$8 \mathrm{El} 24 \%$ de las empresas que no practican VC señalan que un facilitador de su puesta en marcha sería el apoyo de la dirección de la empresa. 
ejercerlo o no. Este hallazgo debe destacarse debido a que estamos ante uno de los factores menos reconocidos por la literatura.

Así pues, la práctica de VC aconseja unas características organizativas particulares centradas en los principios de adaptabilidad a los cambios del entorno, delegación, comunicación interna fluida y personal que gestione las actividades de voluntariado ${ }^{9}$. Todos estos facilitadores muestran una influencia igual o similar.

\section{FACTOR J}

Las respuestas recibidas (tabla 4, gráfico 12 -Anexo-) nos dibujan la intensidad con la que las empresas de la muestra practican VC. Alcanzan una calificación próxima al sobresaliente - a excepción de J4-, y lo hacen así en su promoción y apoyo al voluntariado (J1), en la aplicación en el número de programas completos de voluntariado (J2), y en el grado de participación de sus empleados $y$ directivos en los mismos (J3). No en vano representaban empresas señaladas como las de mejor VC de nuestro país. Esto último nos permitía identificar los parámetros clave de una gran empresa española que pretenda implementar un voluntariado de alto nivel.

Los programas de voluntariado están a menudo considerados como actividades deseadas por los empleados más que como iniciativas estratégicas para la empresa. No obstante, los resultados obtenidos ${ }^{10}$, y pese a no constituir objetivo de nuestro estudio, sugieren un planteamiento estratégico de $\mathrm{VC}$ por parte de las empresas, es decir, supera la visión receptiva recogida en el modelo de Porter y Kramer (2006). Esto implica un VC muy comprometido en el sentido de estar orientado a una mejora de la cadena de valor y de la competitividad a largo plazo de la empresa. La competitividad es un concepto multidimensional pero no disponemos de ninguna escala de factores generalmente aceptada. Basándonos en las ofrecidas por Rubio y Aragón, (2002), Ruiz (2000), Huerta (2002) y Marín y Rubio (2008), podemos corroborar esta mejora competitiva que las empresas de la muestra alcanzarían en virtud de que perciben que el VC aporta beneficios asociados con los recursos humanos - los empleados en un lugar preferente-, la calidad de gestión, la innovación, la orientación al cliente y la mejora de su reputación. La necesidad de contar con el apoyo real de la Alta Dirección subraya más esta perspectiva estratégica aplicada a su voluntariado.

9 No se menciona que deba ser contratado expresamente para ello. De hecho, no parece que consideren la contratación obligatoria dado el valor otorgado a F1.

10 Esto de acuerdo con la evidencia de Basil et alii (2011) de que las empresas grandes apoyan un VC más formalizado y estratégico que las pequeñas empresas. 


\section{Conclusiones}

El presente estudio sugiere que podemos confirmar los factores teóricamente influyentes en la realización de acciones de voluntariado por parte de las empresas. Esto es, las empresas de la muestra perciben con nitidez que:

- La práctica de VC reporta ventajas a los trabajadores, las cuales se trasladan directa o indirectamente a la empresa.

- El VC permite mejorar la imagen y reputación de la empresa, especialmente su imagen de empresa con RSE e innovadora.

- Las actividades de VC aumentan la calidad de gestión de la empresa, entendida como sinónimo de gestión de los agentes implicados en la empresa, sobre todo en lo que se refiere a la satisfacción que produce en los empleados y la comunidad/sociedad.

- El VC mejora las relaciones de la empresa con la comunidad/sociedad en el sentido de que permite crear un entorno estable y saludable de la comunidad, mejora las relaciones de la empresa con esta última así como con las administraciones públicas.

- La disponibilidad de recursos financieros en la empresa marca su actuación en materia de VC, pero en menor medida que otros factores

- El perfil de los trabajadores - con pretensiones de promoción interna, implicados en su entorno y comprometidos con la empresa-, directivos - compromiso sincero de la Alta Dirección y restantes niveles directivos con el compromiso social de la empresa y sus empleados-y, en menor medida, socios - apoyo a la solidaridad social de la empresa y sus empleados-, condiciona la realización de voluntariado por parte de la empresa. Concretamente, estos perfiles relanzan el VC.

- Las características de la estructura organizativa de la empresa se consideran como un factor relevante a la hora de practicar VC, en el sentido de que una estructura flexible - fácilmente adaptable a los cambios del entorno-, con una política de delegaciones de los directivos en directivos inferiores, una comunicación interna fluida en la empresa y la existencia de personal dedicado a gestionar el VC, impulsan las prácticas de VC.

El objetivo más valorado por las empresas a la hora de ejercer voluntariado es el hecho de que la empresa sea considerada ecosocialmente responsable, es decir, que aplica RSE; se confirma así la consideración del VC como un instrumento eficaz de RSE, apuntado ya por investigaciones previas. En un segundo plano, muy próximo, las razones esgrimidas para realizar estas iniciativas de voluntaria- 
do aluden directamente a sus efectos sobre el bienestar personal de los empleados - en múltiples aspectos - y al vínculo emocional con la empresa que se deriva de ello, así como a las mejoras que produce en el entorno de la empresa y en la consiguiente mejoría de las relaciones que mantiene con él - especialmente con la comunidad y la sociedad-.

Y, como telón de fondo, la percepción de que estas empresas practican un voluntariado estratégico, es decir, orientado a la mejora de la cadena de valor y de la competitividad a largo plazo de la empresa.

Estas evidencias permiten desvelar las claves de un VC de éxito en una gran empresa del país, las que, por un lado, pueden servir de inspiración para la incorporación de voluntariado en las pymes - con la lógica cautela por su menor tamaño- $y$, por otro, orientar a los poderes políticos sobre los aspectos en los que debe incidir para el fomento en el país no ya del VC, sino de un VC de calidad. Esto último adquiere carácter incluso de urgencia debido a que el contexto actual de crisis financiera y económica puede utilizarse como una oportunidad para determinar qué papel se espera que cumpla el voluntariado empresarial en un debilitado estado del bienestar (Ministerio de Sanidad, Política Social e Igualdad, 2011) que puede que se convierta en estructural.

Este trabajo podría ampliarse en el futuro en varias direcciones, tales como la introducción del factor sector o tamaño de la empresa como variables de control, es decir, explorar en qué medida los resultados obtenidos pueden segmentarse $-y$, por tanto, variar - de acuerdo con el sector al que pertenece la empresa y el tamaño de ésta. $\mathrm{Al}$ mismo tiempo, pueden verse mejorados con la aplicación de una metodología que explore más profundamente las relaciones entre factores, $y$ de éstos con nuestra variable objetivo, para lo que podríamos aplicar una metodología causal como son las ecuaciones estructurales. 


\section{Anexo. Gráficos de resultados}

Gráfico 3. Factor A: Beneficios para la empresa relacionados con el personal

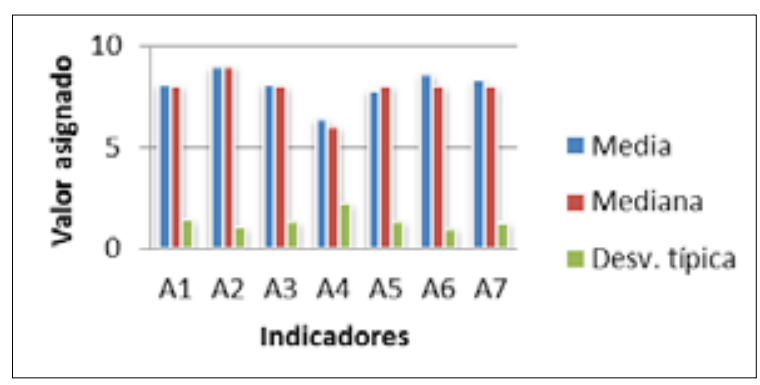

Gráfico 4. Factor B: Beneficios para la empresa relacionados con la mejora de su imagen y reputación

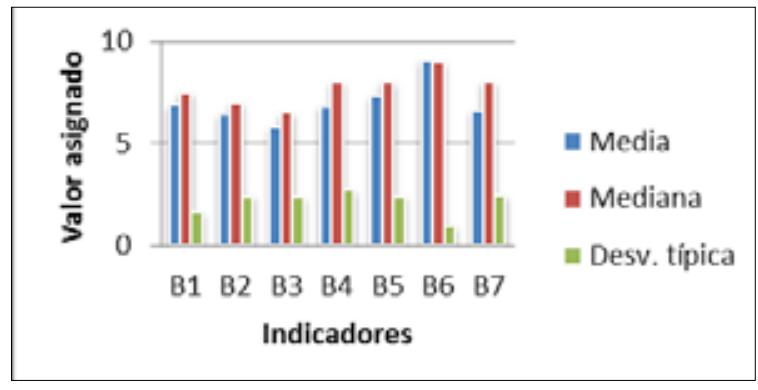

Gráfico 5. Factor C: Beneficios para la empresa relacionados con la mejora de su calidad de gestión

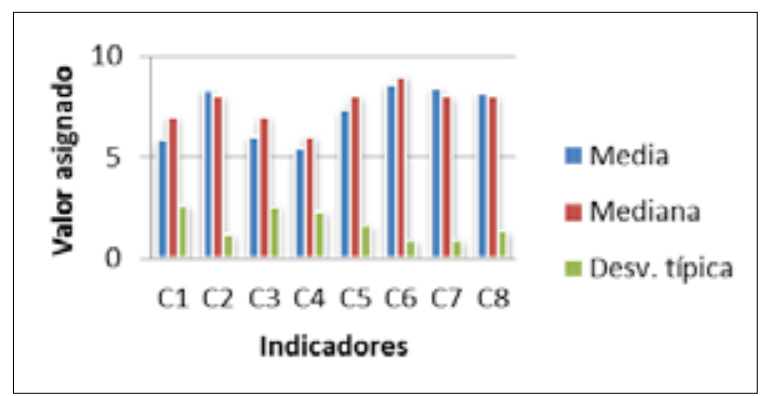


Gráfico 6. Factor D: Beneficios para los trabajadores que redundan en beneficios indirectos para la empresa

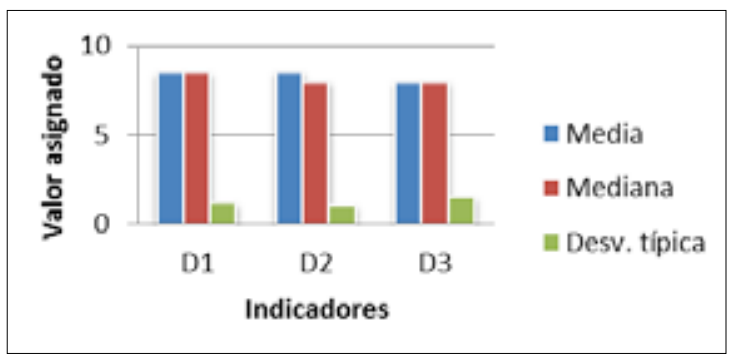

Gráfico 7. Factor Eः Beneficios para la empresa por ser beneficios indirectos para la comunidad

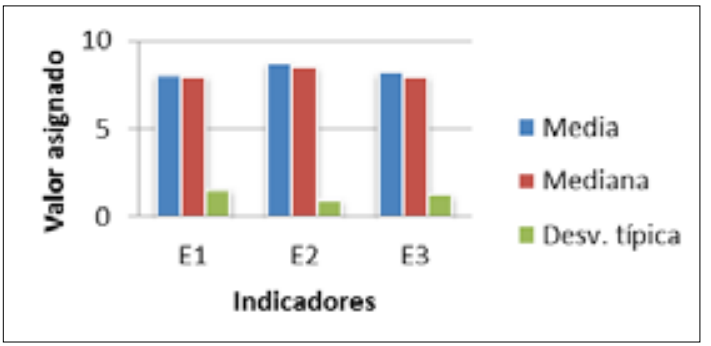

Gráfico 8, Factor F: Recursos

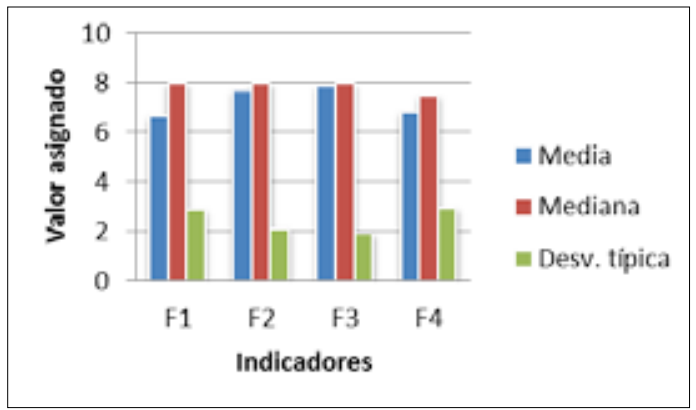


Gráfico 9. Factor Gः Perfil de los trabajadores

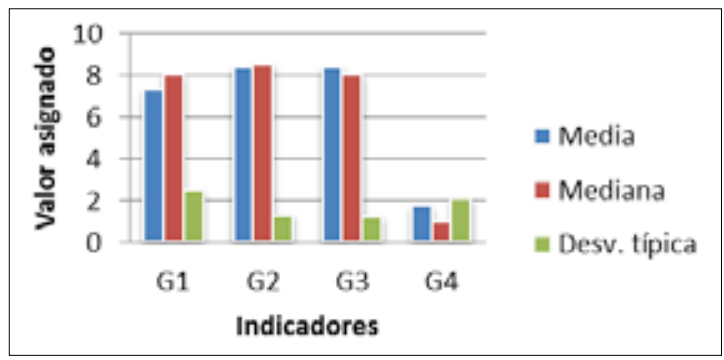

Gráfico 10. Factor H: Perfil de los socios y directivos

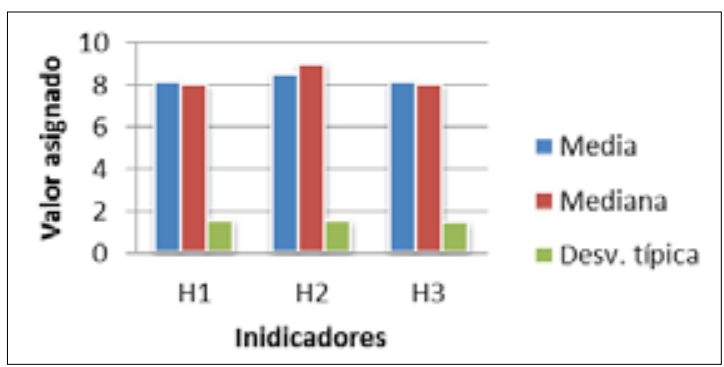

Gráfico 11. Factor Iः Estructura organizativa de la empresa

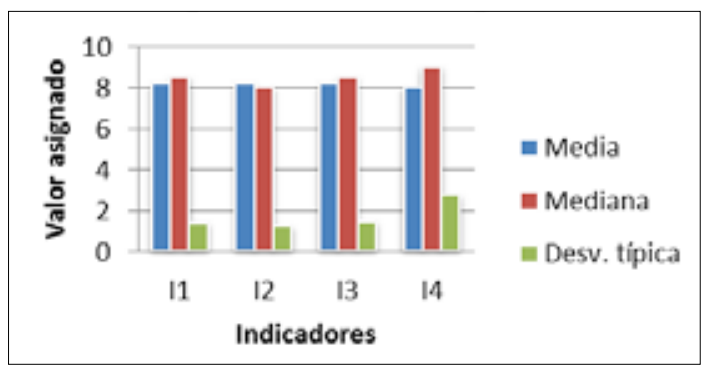

Gráfico 12. Factor Jः Prácticas de voluntariado corporativo en la empresa

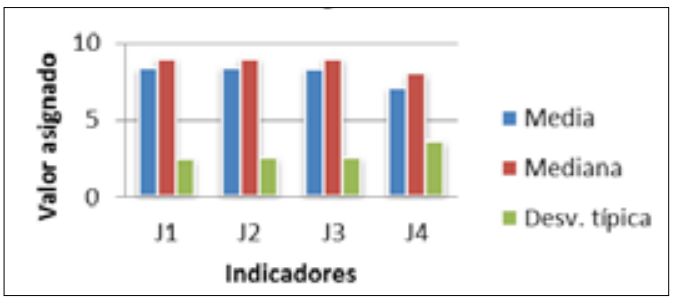




\section{Bibliografía}

AECA (2004). Asociación Española de Contabilidad y Administración de Empresas. Lizcano, J. L. y Moneva, J. M. «Marco Conceptual de la Responsabilidad Social Corporativa». Documento 1 (Responsabilidad Social Corporativa).

AECA (2009). Fernández, M. A. y Muñoz M. J. «Inversión Socialmente Responsable: Estrategias, Instrumentos, Medición y factores de Impulso». Documento 5 (Responsabilidad Social Corporativa).

Albareda, V. L. y Balaguer, F. M. R. (2008). «Contabilidad y Dirección: Responsabilidad Social Corporativa». Working paper.

Aldámiz-Echevarría, C. y Aguirre G. M. (2010), La comunicación de la responsabilidad social de las mayores empresas vascas: un análisis comparativo. Área de Gestión y Organización de Empresas (Sección Ética y Responsabilidad Social). Universidad del País Vasco.

Anuario del Tercer Sector (2010). Anuario del Tercer Sector de Acción Social en España. UE, Caja Madrid y Fundación Luis Vives.

Backhaus, K. B.; Stone, B.A.y Heiner, K.(2002).«Exploring the Relationship Between Corporate Social Performance and Employer Attractiveness». Business E Society, 41: 292-318.

Baiman, S. (1984). «Agency Research in Managerial Accounting: A Survey». En Modern Accounting Research: History, Survey, and Guide (Matтessich, R.): 251-294.

Basil, D.Z. y Erlandson, J. (2008). "Corporate social responsibility website representations: a longitudinal study of internal and external selfpresentations».J Mark Commun, 14 (2): 125-137.

Basil, D.Z;; Runte, M.S.; Easwaramoorthy, M. y Barr, C. (2009). «Company support for employee volunteering: a national survey of companies in Canada». Journal of Business Ethics, 85: 387-398.

Basil, D.Z.; Runte, M.; Basil, M. y Usher, J. (2011). «Company support for employee volunteerism: Does size matter?» Journal of Business Research, 64: 61-66.

Burnes, K. y Gonyea, J. G. (2005). Expanding the boundaries of corporate volunteerism: retirees as a valuable resource. Boston: Boston College Center for Corporate Citizenship.

Campbell, J. L. (2007). "Why Would Corporations Behave in Socially Responsible Ways? An Institutional Theory of Corporate Social Responsibility». Academy of Management Review, 32 (3): 946-967. 
Carrol, P. B. (1990). «Chivas Regal Report on working Americans: emerging values for the 1990s». Wall Street Journal (August 10), B6.

Caudron, S. (1994). «Volunteer Efforts Offer Low-Cost Training Options». Personnel Journal, 73: 38-44.

CExS (2010). Estudio sobre el estado del voluntariado corporativo en España. Club de Excelencia en Sostenibilidad y Fundación Adecco. En línea: <http://www. clubsostenibilidad.org/f_publicaciones/voluntariado.pdf>

Comisión Europea (2011a). COM(2011) 381. «Estrategia renovada de la UE para 2011-2014 sobre la responsabilidad social de las empresas».

- (2011b).COM (2011) 568. «Comunicación sobre políticas de la UE y voluntariado: Reconocimiento y fomento de actividades voluntarias transfronterizas en la UE».

Cone/Roper (1999). Cause related trends report. Boston: Cone Inc.; New York: Roper Starch Worldwide Inc.

DAVIDson, J. (1994). «The case for corporate cooperation in community affairs». Business and Society Review, 90: 29-30.

DÁvila, C. (2010). «Motivaciones personales en voluntariado corporativo». Revista de Responsabilidad Social de la Empresa, 6.

De Gilder, D.; Schuyt, T.N.M. y Breedijk, M. (2005). «Effects of an employee volunteering program on the work force: the ABN-AMRO case». Journal of Business Ethics, 61 (2): 143-152.

Deloitte (2007). «Deloitte Volunteer Impact Survey. Survey data GenY». En línea: <http://ebookbrowse.com/2007-deloitte-volunteer-impact-surveydata-geny-pdf-d47232486>

- (2010). «Deloitte Volunteer Impact Survey. Executive Summary». En línea: <http://www.deloitte.com/assets/Dcom-UnitedStates/Local\%20 Assets/Documents/us_2010DeloitteVolunteerIMPACTSurvey_ ExecutiveSummary_043010.pdf>

- (2011). «Deloitte Volunteer Impact Survey. Executive Summary.» En línea: <http://www.deloitte.com/assets/Dcom-UnitedStates/Local\%20 Assets/Documents/us_2011DeloitteVolunteerIMPACTSurvey_ ExecutiveSummary_060311.pdf>

Den Hond, F., de Bakker, F. G. A. y Neergaard, P. (2007). «Introduction to managing corporate socialresponsibility in action: Talking, doing and measuring». En Den Hond, F., De Bakker, F. G. A. y Neergaard, P. (eds.): Managing Corporate Social Responsibility in Action: Talking, Doing and Measuring. Aldershot, Hampshire, England: Ashgate Publishing: 1-12. 
DiMaggio, P. y Powell, W. (1983). «The iron cage revisited: institutional isomorphism and collective rationality in organizational fields». American Sociological Review, 23: 111-136.

Dowell, G.; Hart, S. y Yeung, B. (2000). «Do corporate global environmental standards create or destroy market value». Management Science, 46 (8): 1059-1074.

Eurobarómetro (2008). «Eurobarómetro 69 Primavera 2008. Valores de los Europeos». En Ministerio de Sanidad, Política Social e Igualdad (2011): Diagnóstico de la situación del voluntariado de acción social en España. Informes, Estudios e Investigación 2011. En línea: <http://www.msc.es/ politicaSocial/ongVoluntariado/docs/diagnosticoSituacionVoluntariado. pdf $>$

Finney, M. (1997). «Operations that build smiles, confidence, skills and community goodwill». HR Magazine, 42 (4): 110-116.

Foro de Reputación Corporativa (2006). Introducción a la reputación corporativa. Madrid (diciembre).

- (2012). <http //www.corporateexcellence.org/index.php/web>

Freeman, R. E. (1984). Strategic Management: a Stakeholder Approach. Pitman Publishing.

Fundación Seres (2011). Diagnóstico para un voluntariado eficaz. Manual Práctico. IE Business School. En línea: <http://www.compromisorse.com/ upload/estudios/000/161/diagnostico_voluntariado_final_2pantalla.pdf> Geroy, G.D.; Wright, P.C. y Jасовy, L. (2000). «Toward a conceptual framework of employee volunteerism: an aid for the human resource manager». Management Decision, 38 (4): 280-287.

González, A.M.; Negueruela, M.; Dávila, M.C. (2008). Voluntariado apoyado por la empresa: Guía para entidades de acción social. Madrid: Dirección General de Voluntariado y Promoción Social. Comunidad de Madrid.

Graff, L. (2004). Making a Business Case for Employer-Supported Volunteerism. Ottawa: Volunteer Canada.

Grant, R. M. (1996). Dirección Estratégica. Conceptos, Técnicas y Aplicaciones. Madrid: Civitas.+

Guardia, R;; Vallés, I; González, M*; Fernández, D. y Serrano, E. (2006). Guía para Promover el Voluntariado desde la Empresa. Fundación La Caixa. Barcelona.

Guerra, A. (2006). Implicación del Sistema Financiero en la Empresa Extremeña. Cáceres: Caja de Ahorros de Extremadura. 
Hess, D*; Rogovsky, N. y Dunfree, T. W. (2002). "The next wave of corporate community involvement: corporate social initiatives». California Managemente Review, 44: 110-125.

Hoare, S. (2004). «Social Workers». Human Resourcesः 40-43.

Huerta, E. (2002). Los desafíos de la competitividad. La innovación organizativa y tecnológica en la empresa española. Bilbao: Fundación BBVA.

Indice del Monitor Español de Reputación Corporativa - Merco (2011). En línea: <http://www.merco.info/es/posts/125>

Lemonche, P. (2011). Voluntariado corporativo. Un puente de colaboración entre la empresa y la sociedad. Forética. En línea: <http‡//www.foretica.org/biblioteca/cuadernos-foretica/doc_details/315-17-voluntariado-corporativo-unpuente-de-colaboracion-entre-la-empresa-y-la-sociedad?lang=es $>$

LEón, Ma J. (2010). El voluntariado corporativo: teoría y práctica para empresas con conciencia social. GTZ Cercapaz. Proyecto Sector Privado y Paz en Manizales. Hito 3: Voluntariado Corporativo.

Lewin, D. (1991). Community involvement, employee morale, y business performance. IBM Worldwise Social Responsibility Conference.

Lewis, A. y Mackenzie, C. (2000). «Morals, Money, Ethical Investing and Economic Psychology». Human Relations, 53(2): 179-191.

Maignan, I.; Ferrell, O.C. y Hult, G.T.M. (1999). «Corporate citizenship: cultural antecedents and business benefits». Academy of Marketing Science. Journal, 27 (4): 455-469.

Margolis, J.D. y Walsh, J.P. (2001). People and profits? The search for a link between a company's social and financial performance. Mahwah NJ: Lawrence Erlbaum Associates.

Marín, J. L. y Rubio, A. (2008). «La responsabilidad social corporativa como determinante del éxito competitivo: un análisis empírico». Revista Europea de Dirección y Economía de la Empresa, 17 (3): 27-42.

Meijs, L.C.P.M. y Roza, M.L. (2010). «The effects of a corporate community program; what is it for the employee and the organization?»Volunteering Counts: A volunteering research conference. (1-2 marzo, Manchester).

Miller, W. H. (1997). «Volunteerism: A New Strategic Tool». Industry Week, 1: $13-16$.

Ministerio de Sanidad, Política Social e Igualdad (2010). Estrategia Estatal del Voluntariado (2010-2014). Diciembre, 2010.

- (2011). Diagnóstico de la situación del voluntariado de acción social en España. Informes, Estudios e Investigación 2011. En línea: <http://www.msc.es/ 
politicaSocial/ongVoluntariado/docs/diagnosticoSituacionVoluntariado. pdf $>$

Montanés, P. y Olier, E. (2006). Corporate Governance Intelligence. Desarrollando la Corporación en Web. Madrid: Pearson Educación.

Muthuri, J. N.; Matten, D. y Moon, J. (2009). «Employee volunteering and social capital: contributions to corporate social responsibility». British Journal of Management, 20: 75-89.

NCB Spain (2011a), Reasons for volunteering / benefits of volunteering. España.

- (2011b). Volunteering in spanish society. España.

Observatorio de VC (2009). Voluntariado Corporativo en España. En línea: <http://www.observatoriovc.org/wp-content/uploads/2012/10/ INFORME_VC_2009_web.pdf>

- (2010). Voluntariado Corporativo en España. En línea: <http://www. observatoriovc.org/InformeVC2010.pdf>

-(2011). Voluntariado Corporativo en España. En línea: <http://www. observatoriovc.org/wp-content/uploads/2012/10/INFORME_ VC_2011_web.pdf>

Observatorio del Tercer Sector (2007). Buenas prácticas en la gestión del voluntariado. Fundación La Caixa. En línea: <http://www. publicacionestecnicas.com/lacaixa/voluntariat/buenas_practicas/files/ publicaciones/buenas_practicas/es/buenas_practicas.pdf>

Orlitzky, M.; Schmidt, F. L. y Rynes, S. L. (2003). «Corporate social and financial performance: a meta-Analysis». Organization Studies, 24 (3): 103441.

Pajo, K. y Lee. L. (2011). «Corporate-sponsored volunteering: a work design perspective». Journal of Business Ethics, 99 (3): 467-482.

Peloza, J. y Hassay, D.N. (2006). «Intra-organizational Volunteerism:Good Soldiers, Good Deeds and Good Politics». Journal of Business Ethics, 64 (4): 357-379.

Penrose, E. T. (1959). The Theory of the Growth of the Firm. New York: Wiley.

Peterson, D. K. (2003). «Benefits of participation in corporate volunteer programs: employees' perceptions». Personnel Review, 33 (6): 615-627.

- (2004a). «Recruitment strategies for encouraging participatioin in corporate volunteer programs». Journal of Business Ethics, 49 (4):371-386.

- (2004b). «Benefits of participation in corporate volunteer programs: employees' perceptions». Personnel Review, 33 (6): 615-627.

Points of Light (1999). Points of Light Foundation and Allstate Foundation. The corporate volunteer program as a strategic resource: the link grows stronger. En 
línea: <http://academic,regis.edu/volunteer/specialcollection/SpecFiles/ grows_stronger.pdf $>$

Points of Light (2000). Points of Light Foundation and Allstate Foundation. The Corporate Volunteer Program as a Strategic Resource: The Link Grows Stronger. Washington, D.C.: The Points of Light Foundation.

Porter, M. (1982). Estrategia Competitiva. México: C.E.C.S.A.

- and Kramer, M. (2002). "The Competitive Advantage of Corporate Philanthropy». Harvard Business Review, 80: 57-68.

- (2006). «Strategy and society: the link between competitive advantage and corporate social responsibility». Harvard Business Review, 84 (12): 78-92.

Rochlin, S.A.y Christoffer, B. (2000). Making the Business Case. Determining the Value of Corporate Community Involvement. Boston: The Center for Corporate Citizenship at Boston College.

Rodríguez, F. y García, J. (2011). «Responsabilidad social corporativa y gobierno corporativo: impacto de la reputación de las compañías del sector automovilístico y la satisfacción al cliente». Revista de Responsabilidad Social de la Empresa, 7.

Rubio, A. y Aragón, A. (2002). «Factores explicativos del éxito competitivo. Un estudio empírico en la pyme». Cuadernos de Gestión, 2 (1): 49-63.

Ruiz, J. (coord.) (2000). Competitividad Regional y Recursos Intangibles. Cádiz: Servicio de Publicaciones de la Universidad de Cádiz.

Rumelt, R. (1984). «Toward a strategic theory of the firm». En Lamb, R. (ed.). Competitive Strategic Management. New York, Englewood Cliffs: PrenticeHall.

Runte, M.; Basil, D.Z.y Runte, R. (2010). «Corporate support for employee volunteerism within canada: A cross-cultural perspective». Journal of Nonprofit and Public Sector Marketing, 22 (4): 247-263.

Sen, S. y Bhattacharya, C. B. (2001). «Does Doing Good always Lead to Doing Better? Consumer reaction to Corporate Social Responsibility». Journal of Marketing Research, 38: 225-244.

SIF (1999). 1999 Report on Responsible Investing Trends in the United States. Social Investment Forum. Washington.

Simon, H. (1962). Tesis doctoral (Universidad de Chicago, 1947): «El comportamiento administrativo. Estudio de los procesos decisorios en la organización administrativa». Madrid: Aguilar.

Smith, C. (2003). «Corporate Social Responsibility: whether or how?». California Management Review, 45 (4): 52-76. 
Stebbins, S. J. (1989). The influence of community service volunteer work on perceptions of jobs satisfaction and organizational commitment among Oregon employees of Pacific Bell Nortwest' Ann Arbor Michigan. Unpublished Doctoral Dissertation.

Steel, K. (1995). «Managing Corporate and Employee Volunteer Programs». En Connors, T. D. (ed.). The Volunteer Management Handbook. New York: Wiley \& Sons.

Stone, B. A. (2001). «Corporate Social Responsibility and Institutional Investment: A Content Analysis-Based Portfolio Screening Model for Socially Responsible Mutual Funds». Business E Society, 40: 112-117.

Suviri, J.I. (2010). «Conceptualización y comparación de distintos modelos de evaluación de la reputación corporativa». Revista de la Responsabilidad Social de la Empresa, 4.

Telefónica (2011). Informe de Sostenibilidad y Responsabilidad Corporativa 2011. Telefónica España. http://info.telefonica.es/es/corporate_responsibility/ pdf/informe_sostyrc_tespana_2011.pdf

Tuffrey, M. (1998). Valuing employee community involvement. London: The Corporate Citizenship Company.

- (2003). Good Companies Better Employees: How Community Involvement and Good Corporate Citizenship Can Enhance Morale, Motivation, Commitment and Performance. London: Corporate Citizenship Company.

VArgas, J.G. (2005). «Análisis de fundamentos de la Teoría Institucional». Revista Digital Universitaria, 6 (8): 1-21. En línea: <http://www.revista. unam.mx/vol.6/num8/art84/ago_art84.pdf>

Vogel, D. (2005). The Market for Virtue: The Potential and Limits of Corporate Social Responsibility. Washington: Brookings Institution Press.

Waddock, S.A. y Graves, S.B. (1997). «The corporate social performancefinancial performance link». Strategic Management Journal, 18 (4):303-319.

Wild, C. (1993). «Corporate Volunteer Programs: Benefits to Business». The Conference Board Report, 1029. Nueva York. 